ISSN: 2174-5609

DOI. https://doi.org/10.14198/INTURI2022.23.16

Cita bibliográfica: Taveras, J.M., Orgaz Agüera, F.y Moral Cuadra, S. (2022). El apoyo de los residentes al desarrollo del agroturismo en el noroeste de la República Dominicana. Investigaciones Turísticas (23), pp. 360378. https://doi.org/10.14198/INTURI2022.23.16

\title{
El apoyo de los residentes al desarrollo del agroturismo en el noroeste de la República Dominicana
}

Resident support for the development of agritourism in the northwest of the Dominican Republic

José Manuel Taveras (iD) Universidad Tecnológica de Santiago, República Dominicana josemanueltaveras@gmail.com

Francisco Orgaz-Agüera (iD) Universidad Tecnológica de Santiago, República Dominicana franorgaz@utesa.edu

Salvador Moral-Cuadra (iD), Universidad de Córdoba, España 162mocus@uco.es

\section{RESUMEN}

Este estudio aborda los antecedentes que pueden influir en el apoyo al desarrollo del agroturismo. Se aplicó un cuestionario a 560 residentes de la región noroeste de la República Dominicana. Esta zona destaca por la agroindustria y tiene índices de pobreza altos. Usando modelos de ecuaciones estructurales, se ha comprobado que el apego a la comunidad y las actitudes hacia el turismo y el turista son antecedentes del apoyo al desarrollo del agroturismo. También se ha evidenciado la importancia del conocimiento sobre turismo por parte de los residentes para confiar en las políticas turísticas públicas.

Palabras clave: Agroturismo; apego a la comunidad; actitudes; apoyo al desarrollo del agroturismo.

\section{ABSTRACT}

This study addresses the antecedents that may influence the support for the development of agrotourism. A questionnaire was conducted among 560 residents of the northwest region of the Dominican Republic. This area stands out for its agribusiness and has high poverty rates. Using structural equation models, it has been proven that attachment to the community and attitudes towards tourism and tourists are antecedents of support for the development of agrotourism. The importance of residents having a knowledge of tourism in order to ensure trust in public tourism policies has also been evidenced.

Keywords: Agrotourism; attachment to the community; attitudes; support for the development of agrotourism. 


\section{INTRODUCCIÓN}

El turismo es una de las principales actividades económicas a nivel mundial (Bianchi, 2018), si bien, en 2020 ha sufrido uno de los mayores retrocesos de su historia por motivos del coronavirus (Williams, 2020). Según datos de la Organización Mundial del Turismo (OMT, 2020), los destinos recibieron un $70 \%$ menos de turistas internacionales respecto al año 2019, con una pérdida de ingresos de 935.000 millones de dólares estadounidenses. Estos datos se deben a que la industria turística tuvo que dejar de operar en el mundo, restringiéndose los viajes internacionales (Williams, 2020). Aunque todos los países del mundo se han visto afectados, cabe resaltar que estos datos son más negativos para los países en desarrollo, puesto que en muchos de ellos se había demostrado que el turismo se relaciona con la reducción de la pobreza (Scheyvens, 2007; Winters et al., 2013).

En República Dominicana, el turismo de sol y playa se ha constituido como una de las principales actividades económicas (Real-Aquino, 2014). Destacan las regiones de Punta Cana, Boca Chica-Juan Dolio y Puerto Plata, donde se ubican, además, los principales aeropuertos internacionales del país, que reciben casi el $80 \%$ de los visitantes que llegan a República Dominicana. En 2019, República Dominicana registró 6.446 .036 turistas internacionales, consolidándose como uno de los principales destinos del Caribe, si bien, se había reducido la cifra de turistas un 1,9\% respecto a 2018 (Banco Central, 2020a). Con la crisis del coronavirus, República Dominicana se ha visto muy afectada, con una gran reducción de visitantes internacionales, lo que ha generado, por un lado, el cierre de muchos complejos hoteleros y de empresas de turismo activo y, por otro lado, el aumento del desempleo de trabajadores del sector turístico (Agencia EFE, 2020).

Dada la crisis actual del turismo debido al coronavirus, es necesario buscar nuevas formas de turismo, enfocándose en actividades en contacto con la naturaleza y que puedan ser atractivas para la demanda turística nacional e internacional. Una de estas actividades puede ser promovida por el agroturismo. De acuerdo con Cors (2020), el agroturismo ha sido analizado por diversos investigadores y académicos, y ha sido incluido como parte del turismo rural (Clarke, 1999; Pulido, 2008). Así, se ha concebido el agroturismo como aquellas actividades turísticas que son promovidas por los agricultores (Di Domenico y Miller, 2012; Tew y Barbieri, 2012), donde se integran aspectos agrarios como los productos, procedimientos, espacios, productores, cultura y valores (Durrande-Moreau et al., 2017).

En República Dominicana, el sector agropecuario es uno de los más importantes, aportando un 4,1\% al Producto Interior Bruto del país (Banco Central, 2020b). Este sector se encuentra, principalmente, en la región noroeste de la República Dominicana. Esta región está conformada por las provincias de Dajabón, Monte Cristi, Santiago Rodríguez y Valverde. Aunque la actividad turística es uno de los principales motores del país, el turismo en esta región es muy bajo y actualmente los organismos nacionales no han recogido datos del impacto de dicha actividad en esta región. Si bien, esta zona cuenta con recursos turísticos potenciales para desarrollar el agroturismo y otras actividades turísticas de índole rural, gastronómicas y culturales (Orgaz-Agüera y López-Guzmán, 2015; Moral Cuadra et al., 2018).

Un estudio realizado recientemente por Oviedo-García et al. (2019) indicó que los ingresos por turismo de sol y playa recibidos en República Dominicana no habían contribuido a reducir la desigualdad en la distribución de la riqueza. Los resultados de este estudio concluyeron que el número de pobres no solo aumenta en República Dominicana, sino que 
los que siguen siendo pobres se vuelven más pobres como consecuencia de la actividad turística. Esto se debe a que la mayoría de los ingresos por turismo recaen en grandes empresas multinacionales que, a su vez, proporcionan sueldos bajos a sus empleados (OviedoGarcía et al., 2019).

Por tanto, es necesitario establecer estrategias para potenciar los beneficios por turismo a través de acciones encaminadas a impulsar empresas turísticas locales $y$, específicamente, en territorios con potencial para el turismo y con menos oportunidades económicas para los residentes. De esta manera, las comunidades locales se beneficiarán no solo del empleo directo generado por el turismo sino también del efecto multiplicador de toda la cadena de valor (Job y Paesler, 2013). Así, la participación de los residentes en el turismo es de importancia clave para beneficiar a las comunidades locales a través de la redistribución de ingresos del turismo (Medina-Muñoz et al., 2016). Es decir, la participación de las comunidades locales en el turismo es vital para crear un vínculo entre la actividad turística y la economía local.

Según los antecedentes indicados en los párrafos anteriores, el objetivo de este artículo es analizar la percepción de los residentes y su relación con el apoyo al desarrollo del agroturismo sostenible en la región noroeste de la República Dominicana. Se ha seleccionado esta región por tres motivos: en primer lugar, porque la principal actividad económica es el sector agropecuario; en segundo lugar, porque dispone de numerosos atractivos naturales y culturales para potenciar el desarrollo del turismo; $y$, en tercer lugar, porque esta región cuenta con un $19,7 \%$ de su población en pobreza, estando un $2,8 \%$ en pobreza extrema (ONE, 2020a). La intención de este artículo es obtener evidencias empíricas sobre el apoyo de sus residentes hacia el desarrollo del agroturismo, lo que puede ayudar al Gobierno Central y Provincial para desarrollar estrategias de turismo en esta región dominicana.

\section{REVISIÓN DE LA LITERATURA}

La revisión de la literatura se ha dividido en dos partes. En primer lugar, se analizan tres constructos (apego a la comunidad, actitud hacia el turismo y actitud hacia los turistas) y su relación con el apoyo hacia el desarrollo del agroturismo. En segundo lugar, se estudia el constructo de conocimiento sobre turismo y como incide en el constructo confianza de los residentes en el gobierno local y, a su vez, como repercute esta variable en el apoyo hacia el desarrollo del agroturismo. Se han seleccionado estos constructos porque ya se conoce su utilidad para valorar el apoyo de los residentes al turismo a través de las percepciones de la comunidad local (Nunkoo, 2015; Eusébio et al., 2018; Martín et al., 2018; Rasoolimanesh et al., 2019).

Lo relevante de este artículo es que se propone el análisis de la relación de estas variables para el desarrollo de un tipo de turismo específico, en un área en vías de desarrollo, con bajos ingresos por turismo y con altos índices de pobreza. Los constructos propuestos también se analizan porque las percepciones de los residentes sobre los impactos del turismo varían según el tipo de turismo (Bestard y Nada, 2007; Sheldon y Abenoja, 2001; Vargas et al., 2009) y, en este caso, el agroturismo no se ha analizado en la República Dominicana y, por consiguiente, es vital conocer sí las comunidades locales ubicadas en zonas rurales, con potencial para el desarrollo del agroturismo, tienen intención de apoyar el desarrollo de esta actividad socioeconómica. Además, este estudio parte de la idea de que en los territorios ubicados en interiores existe el potencial para implementar nuevos clústeres con la necesidad 
de poner en marcha nuevos productos turísticos innovadores (Cànoves et al., 2020), y uno de ellos puede ser el agroturismo.

\subsection{Apego a la comunidad, actitud hacia el turismo y hacia los turistas y su relación con el apoyo al desarrollo del agroturismo}

El apoyo al desarrollo del turismo por parte de los residentes se relaciona con la participación en las ideas y programas para el desarrollo del turismo local. El apoyo de los residentes tiene impactos favorables en los beneficios económicos de los destinos (López et al. 2018), debido a que ayuda a distribuir los beneficios de forma más equitativa. Por lo tanto, el apoyo de los residentes a los programas de turismo es un factor importante que indica el éxito del desarrollo turístico sostenible (López et al. 2018). Si bien, e independientemente del tipo de turismo que se desee apoyar, es preciso analizar las percepciones de los residentes y su relación con el apoyo al turismo sostenible. En este sentido, el apoyo de los residentes al desarrollo turístico está influenciado por una variedad de factores distintos, pero interconectados (Látková y Vogt 2012; Rasoolimanesh et al., 2015) y, por esta razón, es necesario conocer elementos como el apego a la comunidad, la actitud hacia el turismo y la actitud hacia los turistas.

El apego a la comunidad se describe como la cohesión de los residentes con el destino, incluyendo el impacto, el significado, el sentimiento, los valores intangibles y la conexión que tienen los residentes con el destino (Nicholas et al., 2009; Lee, 2013). Es decir, el apego comunitario es la dependencia que una persona tiene con el lugar donde vive (López et al. 2018). Se ha demostrado que los residentes que tienen un sentido de vínculo con la comunidad ven más beneficios socioeconómicos por turismo que aquellos que tienen un menor vínculo (Lee, 2013). Así, se ha demostrado que el sentido de apego a la comunidad puede ser determinante clave de su apoyo al desarrollo del turismo sostenible (Gannon et al., 2020).

En su estudio, Moghavvemi et al. (2017) sugieren que el sentido de apego a la comunidad de los residentes tiene la capacidad de predecir actitudes sobre el desarrollo turístico, debido al hecho de que los residentes que están fuertemente comprometidos con su comunidad están más involucrados y expuestos a los impactos del turismo. De esta forma, el apego a la comunidad se caracteriza por una simbiosis entre el residente y la comunidad, donde prevalece el orgullo, que a menudo se manifiesta como un deseo de mostrar los aspectos positivos de un destino a los visitantes (Rasoolimanesh et al. 2017). Así, el apego a la comunidad puede estimular el apoyo de los residentes hacia el desarrollo de una industria turística y sus ofertas de servicios asociados dentro de su comunidad local si se diseña e implementa de una manera comprensiva y apropiada (McCool y Martin 1994). En relación con la información anterior, se propone la siguiente hipótesis:

$\mathrm{H}_{1}$ : El apego a la comunidad por parte de los residentes influye positivamente en el apoyo al desarrollo del agroturismo.

La Teoría del Intercambio Social se ha utilizado para analizar la relación existente entre los residentes, el turismo y los turistas (Gursoy y Kendall, 2006; Dyer et al., 2007; Látková y Vogt, 2012; Vargas et al., 2015). Según esta teoría, los turistas y residentes se involucran en un intercambio social con el objetivo de lograr resultados satisfactorios (Sharpley, 2008). En general, si los residentes perciben que los impactos del turismo pueden ser o son positivos, entonces tendrán una actitud positiva hacia el desarrollo turístico en sus comunidades 
(Sharpley, 2014). Así, se ha demostrado que una actitud favorable hacia los turistas o el turismo, por parte de los residentes de una comunidad, fomenta el apoyo de la propia comunidad hacia el desarrollo del turismo sostenible (Dyer et al. 2007, Nunkoo y Gursoy, 2012; Vargas et al., 2015; Wang y Xu, 2015). En este sentido, el estudio de Martín et al. (2018) concluyó que una actitud positiva hacia el turismo y los turistas conducirá a un comportamiento más positivo de apoyo al turismo en las comunidades de acogida. Siguiendo la información anterior, se proponen las siguientes hipótesis:

$\mathrm{H}_{2}$ : La actitud hacia el turismo por parte de los residentes influye de manera positiva en el apoyo al desarrollo del agroturismo.

$\mathrm{H}_{3}$ : La actitud hacia los turistas por parte de los residentes influyen de manera positiva en el apoyo al desarrollo del agroturismo.

\subsection{Conocimiento del turismo, confianza en el gobierno local y su relación con el desarrollo del agroturismo}

El conocimiento de los residentes sobre el turismo es fundamental para la sostenibilidad y la buena gobernanza del sector turístico (Moscardo, 2005). En este sentido, el conocimiento se puede definir como la comprensión de los residentes hacia los problemas del desarrollo turístico y el papel del gobierno local en la industria (Nunkoo y So, 2015). El conocimiento es un recurso importante para los diferentes actores turísticos y determina su posición en una red de intercambio social (Cook et al. 2013). Se ha demostrado que los residentes que tienen conocimientos sobre turismo tienen más probabilidades de informar sobre impactos positivos (Andereck et al., 2005). Nunkoo (2015) indicó que existe una relación significativa entre el conocimiento por turismo y el apoyo hacia el desarrollo del turismo.

La confianza se define como "un estado psicológico que comprende la intención de aceptar la vulnerabilidad basada en expectativas positivas de las intenciones o el comportamiento de otro" (Rousseau et al., 1998:395). En el turismo, si bien el gobierno no puede obligar a las comunidades locales a mostrar actitudes positivas hacia la industria, requiere reacciones favorables de ellas para garantizar el desarrollo sostenible (Nunkoo y So, 2015). La confianza de los residentes en los actores gubernamentales involucrados en la planificación turística condiciona la forma en que reaccionan al apoyo hacia el turismo (Nunkoo y So, 2015). Esto se debe a que la confianza afecta a las actitudes del público hacia las políticas y los resultados del gobierno (Hetherington y Husser, 2012). Se ha demostrado que la confianza de los residentes en las instituciones turísticas gubernamentales predice significativamente las percepciones y el apoyo de los residentes hacia el desarrollo del turismo (Nunkoo y Ramkissoon, 2011). Aunque un gobierno cumple con los atributos de confiabilidad, no significa necesariamente que los ciudadanos posean el conocimiento suficiente para creer que actúan correctamente (Nunkoo y So, 2015). En este sentido, algunos estudios han validado una relación positiva entre el conocimiento de los ciudadanos sobre los procesos gubernamentales y la confianza política (Grimmelikhuijsen, 2012), sin embargo, esta evidencia no se ha analizado en gran medida en los estudios de turismo (Nunkoo y So, 2015). A partir de las informaciones anteriores, se proponen las siguientes hipótesis:

$\mathrm{H}_{4}$ : El conocimiento del turismo por parte de los residentes influye positivamente sobre la confianza en el gobierno local.

$\mathrm{H}_{5}$ : La confianza en gobierno local por parte de los residentes influye de manera positiva en el apoyo al desarrollo del agroturismo. 
El modelo estructural propuesto se presenta en la siguiente figura:

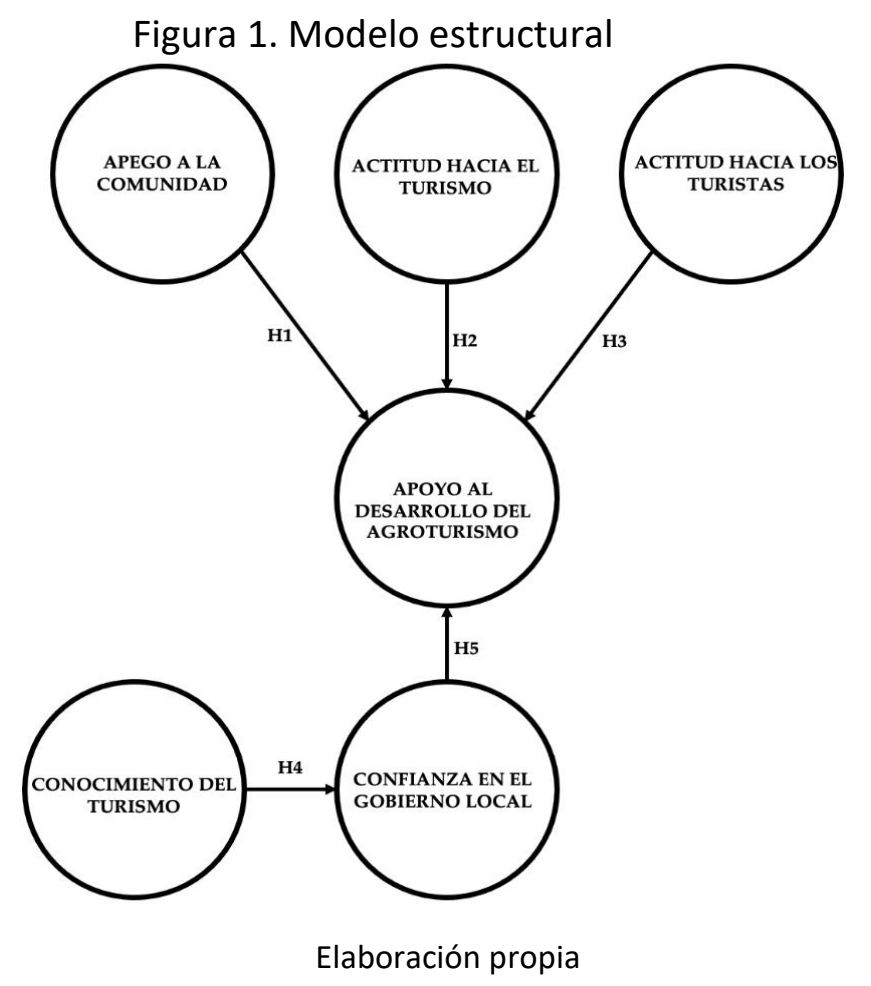

\section{METODOLOGÍA}

\subsection{Localización del área objeto de estudio}

La población objeto de este estudio fueron los residentes locales de la región noroeste de República Dominicana. Dicha región queda conformada por las provincias de Valverde, Monte Cristi, Dajabón y Santiago Rodríguez. La zona objeto de estudio (figura 2) cuenta con un total de 377.307 habitantes (ONE, 2020b), siendo la principal zona agroindustrial del país (Orgaz-Agüera y López-Guzmán, 2015). En esta región el turismo es escaso, si bien, tiene un gran potencial para desarrollar actividades de turismo (Orgaz-Agüera y López-Guzmán, 2015).

Figura 2. Zona objeto de estudio

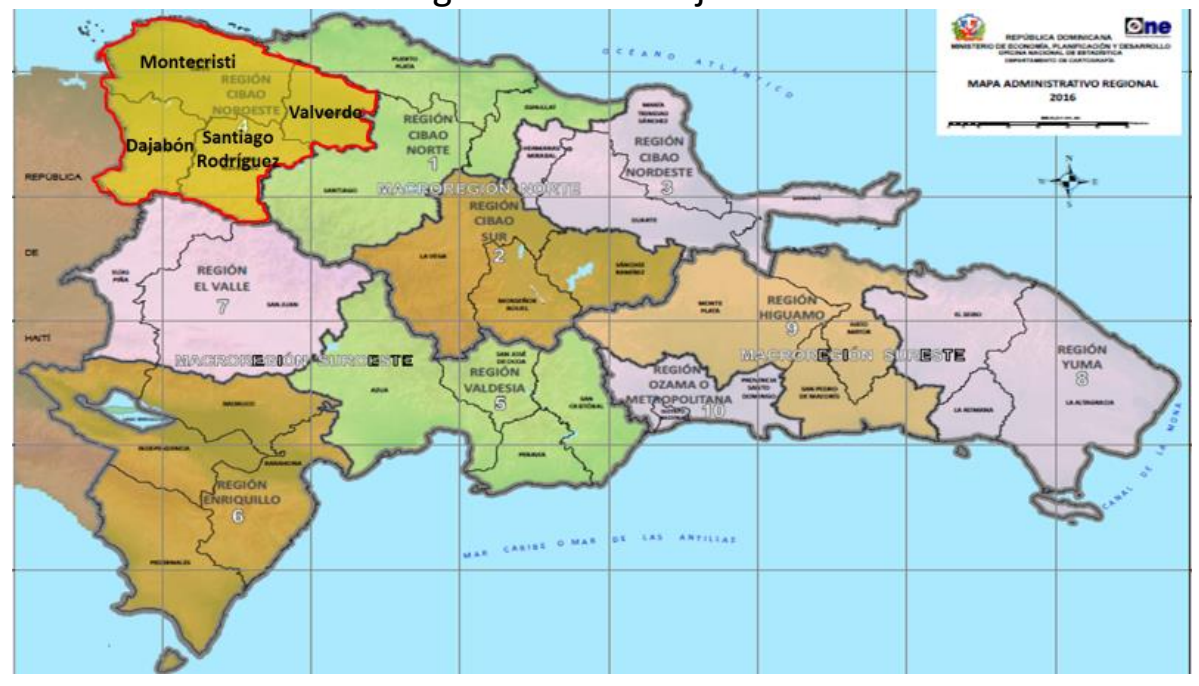

Fuente: ONE (2020b) 


\subsection{Diseño de la encuesta y recolección de datos}

El instrumento empleado se ha basado en un cuestionario debidamente estructurado, donde los aspectos abordados se han realizado respondiendo a una escala de Likert de 5 puntos ( 1 = Nada de acuerdo; 3 = Ni en desacuerdo ni de acuerdo; 5 = Muy de acuerdo). El cuestionario se ha estructurado en tres partes claramente diferenciadas. La primera de ellas abordaba percepciones sobre el turismo, donde los constructos abordados fueron "Actitud hacia el turismo", "Actitud hacia los turistas", "Conocimiento del turismo" y "Confianza en el gobierno local". En el segundo bloque del cuestionario se hacía referencia a la región de procedencia, abordándose cuestiones como el "Apego a la comunidad" y el "Apoyo al desarrollo del agroturismo". Estos constructos han sido seleccionados siguiendo la literatura científica y se muestran, junto a sus ítems, en la tabla 1. Finalmente, en el tercero de los apartados, se presentaron cuestiones relativas al perfil sociodemográfico.

Para la elaboración del cuestionario se ha seguido un proceso basado en cuatro fases. En la primera de las etapas se llevó a cabo una exhaustiva revisión de la literatura existente a través de la cual se determinaron los diferentes constructos que conformarían el modelo. En la segunda de las fases se identificaron los ítems para cada constructo seleccionado. Estos ítems se extrajeron de estudios previos (Nunkoo, 2015; Nunkoo y So, 2016; Eusébio et al., 2018; Martín et al., 2018; Rasoolimanesh et al., 2019). En la tercera de las fases, se tradujeron los ítems al español por una empresa de traducción. Por último, se realizó un pretest de 30 encuestas con el fin de detectar posibles errores de interpretación.

El trabajo de campo fue llevado a cabo entre los meses de octubre 2019 y febrero 2020, distribuyéndose el cuestionario en español, debido a que es la lengua madre de todo el universo poblacional del estudio. El cuestionario era anónimo. El número total de cuestionarios obtenidos fue de 586, si bien, tras un proceso de depuración el total de cuestionarios válidos obtenidos fue de 560 , lo que implica un error muestral del $\pm 4,14 \%$.

\subsection{Perfil sociodemográfico}

En cuanto al perfil sociodemográfico de la muestra, existe casi paridad en relación con el género ( $47,3 \%$ son hombres y $52,7 \%$ son mujeres). Si atendemos a la edad del encuestado, podemos concluir que se trata de personas relativamente jóvenes, puesto que más de dos terceras partes de total se encuentran con una edad por debajo de los 39 años (56,3\% con edad entre 18 y 29 años y un $23 \%$ con una edad entre 30 y 39 años), con un nivel de estudios superior en dos terceras partes del total $(68,3 \%$ con estudios universitarios y solamente un $5,1 \%$ sin ningún tipo de estudios). En cuanto a la provincia de residencia de los encuestados destaca la de Valverde con un 47,9\% del total, seguido de Monte Cristi (20\%), Santiago Rodríguez $(18,6 \%)$ y Dajabón $(13,4 \%)$. El sueldo mensual es inferior a los RD\$10.000 (141€ aproximadamente) en un $40 \%$ de los encuestados, mientras que solamente un $12,8 \%$ de los encuestados declara un salario mensual superior a los $\mathrm{RD} \$ 30.000$ (423€ aproximadamente). Un $80 \%$ de la muestra declara estar trabajando.

\subsection{Análisis de datos}

Para el análisis preliminar de datos (por ejemplo, perfil sociodemográfico o fiabilidad de la escala de medida) se acudió al programa SPSS 24.0, mientras que para el modelo basado en ecuaciones estructurales se utilizó SmartPLS 3.2.8. En este sentido, se ha empleado un modelo basado en mínimos cuadrados parciales, el cual es un método muy común en el campo 
de las ciencias sociales (Hair et al., 2017; Henseler, 2017) y más en concreto en el turismo (Sarstedt et al. 2020; Wang et al., 2020).

A través de SPSS se realizó el análisis de fiabilidad de la escala a través del Alfa de Cronbach, obteniéndole un alfa (a) global de 0,926, lo que implica una fiabilidad de la escala muy por encima de los valores de referencia de 0,7 indicado por autores de referencia (Nunnally y Bernstein, 1994).

Para examinar las relaciones existentes entre constructos a través de ecuaciones estructurales mediante mínimos cuadrados parciales es clave establecer la diferencia entre los compuestos Modo A (constructos reflectivos) y Modo B (constructos formativos). Los constructos Modo A han sido "Conocimiento del turismo", "Confianza en el gobierno local", "Apego a la comunidad" y el "Apoyo al desarrollo del agroturismo", y los constructos Modo B han sido "Actitud hacia el turismo" y "Actitud hacia los turistas". En el caso de los compuestos Modo A, los indicadores son una manifestación del constructo, mientras que los compuestos Modo B son la causa del constructo (Diamantopoulos y Winklhofer, 2001).

\section{RESULTADOS}

Dado el carácter explicativo de la investigación, el análisis se centrará además de la validación del modelo de medida, en el coeficiente de determinación de las variables dependientes, así como la inferencia estadística de las relaciones estructurales o contraste de hipótesis y del tamaño de efecto (Henseler, 2018).

\subsection{Análisis de fiabilidad y validez del modelo de medida}

El análisis a nivel individual de validez y fiabilidad del modelo de medida se lleva a cabo a través del análisis de las cargas factoriales para los compuestos Modo $A$, donde cargas superiores a 0,707 son aceptadas (Ali et al., 2018), si bien, y dado el carácter inicial de esta investigación han sido aceptadas cargas inferiores a 0,707 (Chin, 1998). Para los compuestos Modo $B$, este análisis es llevado a cabo a través de los pesos, donde pesos no significativos, pero con cargas externas asociadas superiores a 0,5 han de mantenerse (Hair et al., 2017). La posible existencia de multicolinealidad entre los indicadores pertenecientes a los compuestos Modo B fue medida a través de la prueba del Factor de Inflación de la Varianza (VIF) (Diamantopoulos y Siguaw, 2006), donde valores de este índice superiores a 3,3 implican problemas de multicolinealidad (Roberts y Thatcher, 2009). En este modelo, todos los indicadores pertenecientes a los compuestos Modo B se han mantenido, mientras que los indicadores con cargas inferiores a 0,707 también se han mantenido ya que su eliminación no suponía una mejora sustancial y significativa del modelo (tabla 1). Tampoco se han identificados problemas de multicolinealidad asociados. 
Tabla 1: Análisis de fiabilidad y validez del modelo de medida

\begin{tabular}{|c|c|c|c|c|c|}
\hline & $\lambda$ & Pesos (Sig.) & VIF & $\rho A$ & AVE \\
\hline $\begin{array}{l}\text { Apego a la Comunidad (AC) } \\
\text { AC1 - Siento que esta región es parte de mí } \\
\text { AC2 - Esta región es muy especial para mí } \\
\text { AC3 - Me identifico fuertemente con esta región } \\
\text { AC4 - Me siento muy conectado con esta región y con las personas } \\
\text { que viven aquí } \\
\text { AC5 - Esta región significa mucho para mí } \\
\text { AC6 - No cambiaría vivir en este lugar por otro lugar } \\
\text { AC8 - Extraño esta región cuando no estoy aquí } \\
\text { AC9 - Tengo sentimientos positivos hacia esta región } \\
\text { AC10 - Tengo un sentimiento de pertenencia por esta región } \\
\text { AC11 - Tengo un apego emocional a esta región, que es muy } \\
\text { especial para mí }\end{array}$ & $\begin{array}{l}0,73 \\
0,79 \\
0,85 \\
0,74 \\
\\
0,75 \\
0,60 \\
0,65 \\
0,72 \\
0,61 \\
0,66\end{array}$ & & & 0,90 & 0,51 \\
\hline $\begin{array}{l}\text { Conocimiento del Turismo (CT) } \\
\text { CT1 - Conozco como el turismo desarrolla mi región } \\
\text { CT2 - Conozco sobre los turistas que visitan mi región } \\
\text { CT3 - Conozco los posibles impactos del turismo } \\
\text { CT4 - Entiendo el rol del gobierno local en el desarrollo del turismo } \\
\text { CT5 - Conozco las diferentes políticas turísticas del gobierno local }\end{array}$ & $\begin{array}{l}0,68 \\
0,76 \\
0,78 \\
0,84 \\
0,83\end{array}$ & & & 0,89 & 0,61 \\
\hline $\begin{array}{l}\text { Confianza en el Gobierno Local (CP) } \\
\text { CP1 - Confío en las decisiones turísticas tomadas por el gobierno } \\
\text { local } \\
\text { CP2 - Confío en el gobierno local } \\
\text { CP3 - El gobierno local está haciendo buen trabajo para el turismo } \\
\text { de la región }\end{array}$ & $\begin{array}{l}0,92 \\
0,93 \\
0,91\end{array}$ & & & 0,91 & 0,84 \\
\hline $\begin{array}{l}\text { Apoyo al Desarrollo del Agroturismo (AD) } \\
\text { AD6 - Me gustaría visitar lugares agroturísticos en mi región } \\
\text { AD7 - Recomiendo los atractivos agroturísticos que existen en mi } \\
\text { región a otras personas } \\
\text { AD9 - Creo que el agroturismo debe fomentarse más en mi región } \\
\text { AD10 - Apoyo el desarrollo del agroturismo } \\
\text { AD11 - Me gustaría que el agroturismo se convirtiera en parte } \\
\text { importante para mi región } \\
\text { AD12 - El gobierno local debe fomentar más el desarrollo del } \\
\text { agroturismo en la región }\end{array}$ & $\begin{array}{l}0,70 \\
0,68 \\
0,72 \\
0,76 \\
0,75 \\
0,70\end{array}$ & & & 0,81 & 0,52 \\
\hline $\begin{array}{l}\text { Actitud hacia el Turismo (AS) } \\
\text { AS1 - El agroturismo generaría beneficios políticos para mi región } \\
\text { AS2 - El agroturismo sería una buena actividad para mi región } \\
\text { AS3 - Me gustaría que el sector agroturismo desempeñe un papel } \\
\text { importante en mi región } \\
\text { AS4 - El agroturismo debe ser fomentado activamente en mi } \\
\text { región }\end{array}$ & $\begin{array}{l}0,56 \\
0,75 \\
0,89 \\
0,81\end{array}$ & $\begin{array}{l}0,18(0,000) \\
0,27(0,000) \\
0,47(0,000) \\
0,34(0,000)\end{array}$ & $\begin{array}{l}1,35 \\
1,67 \\
1,88 \\
1,78\end{array}$ & 1,00 & \\
\hline $\begin{array}{l}\text { Actitud hacia los Turistas (AT) } \\
\text { AT1 - La interacción con los agroturistas que visitarían mi región } \\
\text { sería agradable } \\
\text { AT2 - La interacción con los agroturistas que visitarían mi región } \\
\text { sería placentera }\end{array}$ & $\begin{array}{l}0,89 \\
0,65 \\
0,77\end{array}$ & $\begin{array}{l}0,62(0,000) \\
0,06(0,300) \\
0,43(0,000)\end{array}$ & $\begin{array}{l}1,58 \\
1,61 \\
1,41\end{array}$ & 1,00 & \\
\hline
\end{tabular}




\begin{tabular}{|l|l|l|l|l|}
\hline AT3 - Interactuar con los agroturistas que visitarían mi región sería & 0,60 & $0,12(0,140)$ & 1,40 & \\
divertida & $\begin{array}{l}\text { AT4- La interacción con los agroturistas que visitarían mi región } \\
\text { sería positiva }\end{array}$ & 0,60 \\
\hline
\end{tabular}

Notas: $\lambda$ = cargas factoriales; VIF = Test del Factor de Inflación de la Varianza; $\rho A=$ Fiabilidad Compuesta de Dijkstra-Henseler; AVE: Varianza Extraída Media (Validez convergente)

Elaboración propia

A nivel de constructo, la fiabilidad y validez es medida mediante la fiabilidad compuesta de Dijkstra-Henseler $(\rho \mathrm{A})$, siendo el instrumento que mejor mide la consistencia interna (Dijkstra y Henseler, 2015). Autores como Koufteros (1999) marcan un umbral mínimo de 0,8 para la existencia de una óptima fiabilidad compuesta, situación que es satisfecha en el presente modelo (tabla 2), ya que todos los constructos presentan valores superiores a 0,80 , situándose algunos de ellos por encima o igual a 0,9 (Apego a la comunidad y Confianza en el gobierno local).

La validez convergente y la validez discriminante han presentado valores óptimos en lo que a las pruebas empleadas se refiere. Para la validez convergente (tabla 1 ) se ha empleado la Varianza Extraída Media, donde valores superiores a 0,5 señalan una buena validez convergente del modelo (Fornell y Larcker, 1981). Por otro lado, la validez discriminante (tabla 2) es medida a través de la ratio Heterotrait-Monotrait (Henseler et al., 2015), donde valores inferiores a 0,85 indicarían una buena validez discriminante (Kline, 2011). Tanto la validez convergente como la validez discriminante han arrojado resultados óptimos en este estudio.

Tabla 2: Validez discriminante

\begin{tabular}{|c|c|c|c|}
\hline \multirow{2}{*}{\multicolumn{4}{|c|}{\begin{tabular}{|l} 
Apego a la comunidad (AC) \\
\end{tabular}}} \\
\hline & & & \\
\hline Apoyo al desarrollo del agroturismo (AD) & 0,66 & & \\
\hline Confianza en el gobierno local (CP) & 0,21 & 0,16 & \\
\hline Conocimiento del turismo (CT) & 0,32 & 0,23 & 0,72 \\
\hline
\end{tabular}

\subsection{Análisis del modelo estructural}

Elaboración propia

El modelo estructural es evaluado a través del coeficiente de determinación de las variables dependientes, el tamaño de efecto y la inferencia estadística de las relaciones estructurales, si bien, previamente se ha testado la existencia de colinealidad entre los distintos constructos que conforman el modelo. En este sentido, valores de VIF superiores a 5 indicarían problemas de colinealidad (Hair et al., 2014). No se han identificado ningún tipo de problema asociado a esta anomalía, tal y como puede observarse en la tabla 3.

Tabla 3. Colinealidad del modelo estructural

\begin{tabular}{|l|c|c|}
\hline & Apoyo al desarrollo del agroturismo & Confianza en el gobierno local \\
\hline Actitud hacia el turismo & 1,001 & \\
\hline Actitud hacia los turistas & 1,000 & \\
\hline Apego a la comunidad & 1,002 & \\
\hline Apoyo al desarrollo del agroturismo & & \\
\hline Confianza en el gobierno local & 1,001 & 1,000 \\
\hline Conocimiento del turismo & & \\
\hline
\end{tabular}

Elaboración propia 
El modelo estructural se encuentra ajustado, encontrado un valor de SRMR del modelo saturado de 0,066, encontrándose por debajo de 0,08, valor máximo parta que exista un ajuste del modelo (Hu y Bentler, 1998).

El coeficiente de determinación $\left(R^{2}\right)$ hace referencia a una medida de poder predictivo, indicando la cantidad de varianza de un determinado constructo que es explicado por las variables predictoras, lo que muestra una estrecha relación con la varianza explicada (tabla 4). En este sentido, los constructos "Apoyo al desarrollo del agroturismo" y "Confianza en el gobierno local" presentan unos valores de $\mathrm{R}^{2}$ de 0,42 y 0,45 respectivamente, lo que indican un poder predictivo moderado (Hair et al., 2014). En cuanto a la varianza explicada, la variable "Apego a la comunidad" explica un $25 \%$ de la variabilidad del "Apoyo al desarrollo del agroturismo", mientras que la "Actitud hacia el turismo" y la "Actitud hacia los turistas" explican un $9 \%$ y un $8 \%$ respectivamente de la varianza de la variable "Apoyo al desarrollo del agroturismo". Por otro lado, la variable conocimiento del turismo logra explicar un $45 \%$ de la variabilidad de la variable confianza en el gobierno local.

Tabla 4: Poder predictivo y tamaño de efecto

\begin{tabular}{|l|l|l|l|l|l|}
\hline & $\mathbf{R}^{\mathbf{2}}$ & $\boldsymbol{\beta}$ & Corr. & Var. Expl. & $\mathbf{f}^{\mathbf{2}}$ (p.lim.) \\
\hline Apoyo al desarrollo del agroturismo & $\mathbf{0 , 4 2}$ & & & & \\
H1: Apego a la comunidad & & 0,43 & 0,58 & 0,25 & $0,26(0,000)$ \\
H2: Actitud hacia el turismo & & 0,19 & 0,47 & 0,09 & $0,04(0,02)$ \\
H3: Actitud hacia los turistas & & 0,17 & 0,46 & 0,08 & $0,03(0,06)$ \\
H5: Confianza en el gobierno local & & 0,03 & 0,14 & 0,004 & $0,00(0,330)$ \\
\hline Confianza en el gobierno local & $\mathbf{0 , 4 5}$ & & & & \\
H4: Conocimiento del turismo & & 0,67 & 0,67 & 0,45 & $0,83(0,000)$ \\
\hline
\end{tabular}

Elaboración propia

El tamaño de efecto $\left(\mathrm{f}^{2}\right)$ pone de manifiesto un efecto grande del "Conocimiento del turismo" sobre la "Confianza en el gobierno local", mientras que el "Apego a la comunidad" genera un efecto moderado sobre el "Apoyo al desarrollo del agroturismo". Tanto la "Actitud hacia el turismo" como la "Actitud hacia los turistas" generan un efecto pequeño sobre el "Apoyo al desarrollo del agroturismo", si bien, el efecto de la "Actitud hacia los turistas" no es significativo.

La inferencia estadística de las relaciones estructurales (tabla 5) se han analizado a través de un bootstrapping de 10000 muestras (Streukens y Leroi-Werelds, 2016), obteniéndose la significación estadística a través de la t de student e intervalos de confianza (Henseler, Ringle y Sinkovics, 2009).

Tabla 5: Contraste de hipótesis

\begin{tabular}{|c|c|c|c|c|c|c|}
\hline & \multirow{2}{*}{ Coef. Path } & \multirow{2}{*}{$t$} & \multirow{2}{*}{ P. valor } & \multicolumn{2}{|c|}{ IC (95\%) } & \multirow{2}{*}{ ¿Soporta? } \\
\hline & & & & $5 \%$ & $95 \%$ & \\
\hline$A C \rightarrow A D$ & $0,43 * * *$ & 11,51 & 0,000 & 0,37 & 0,49 & Sí \\
\hline$A S \rightarrow A D$ & $0,19 * * *$ & 4,56 & 0,000 & 0,13 & 0,27 & Sí \\
\hline$A T \rightarrow A D$ & $0,17^{* * *}$ & 3,64 & 0,000 & 0,10 & 0,25 & Sí \\
\hline $\mathrm{CT} \rightarrow \mathrm{CP}$ & $0,67^{* * *}$ & 28,42 & 0,000 & 0,64 & 0,71 & Sí \\
\hline$C P \rightarrow A D$ & $0,03^{\mathrm{NS}}$ & 1,10 & 0,130 & 0,02 & 0,09 & No \\
\hline
\end{tabular}

Notas: $A C=$ Apego a la comunidad; $A D=$ Apoyo al desarrollo del agroturismo; $C P$ = Confianza en el gobierno local; $\mathrm{CT}=$ Conocimiento del turismo; $\mathrm{AS}=$ Actitud hacia el turismo; $\mathrm{AT}=$ Actitud hacia los turistas. ${ }^{*} \mathrm{p}<0,05 ; * * \mathrm{p}<$ 0,$01 ;{ }^{* *} \mathrm{p}<0,001 ; \mathrm{NS}$ : no significante (una cola) $\mathrm{t}(0,05 ; 9999)=1,645 ; \mathrm{t}(0,01 ; 9999)=2,327 ; \mathrm{t}(0,001 ; 9999)=$ 3,092 .

\section{Elaboración propia}


Los resultados obtenidos en la tabla anterior ponen de manifiesto la influencia positiva del apego a la comunidad, la actitud hacia el turismo y la actitud hacia los turistas en el apoyo al desarrollo del turismo. También se pone de manifiesta la influencia positiva del conocimiento del turismo sobre la confianza en el gobierno local. Por otro lado, no se ha soportado aquella hipótesis que indicaba una influencia positiva de la confianza en el gobierno local sobre el apoyo al desarrollo del turismo.

El modelo estructural final se presenta en la figura 3.

Figura 3. Modelo estructural final

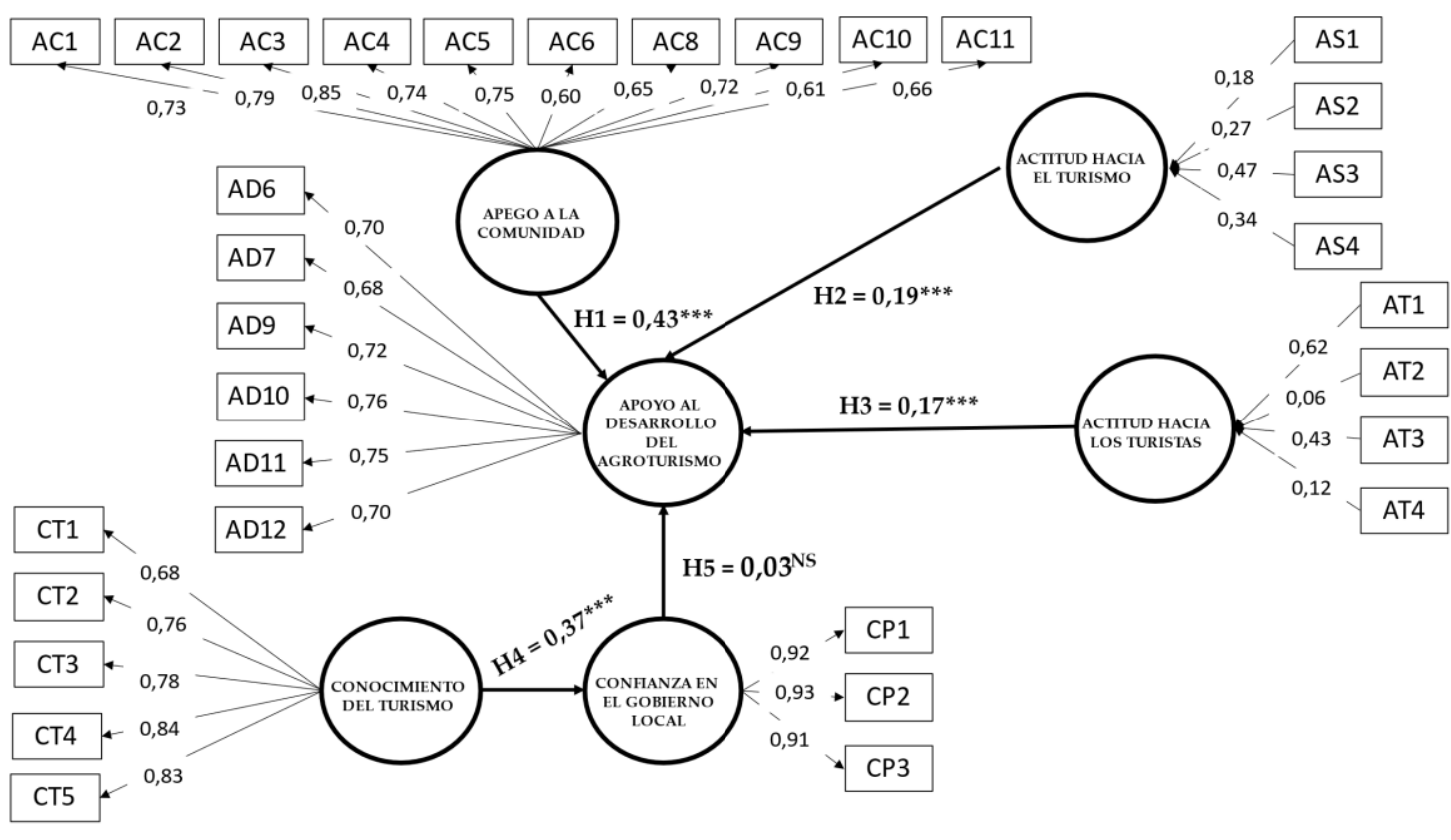

Elaboración propia

\section{DISCUSIÓN DE LOS RESULTADOS Y CONCLUSIONES}

El turismo y la agroindustria forman parte de los sectores socioeconómicos más importantes para la República Dominicana. El turismo de sol y playa destaca sobre todo en las zonas del este y norte del país. Por su parte, la agroindustria, aunque se observa por toda la geografía dominicana, destaca sobre todo en la región noroeste (provincias de Dajabón, Monte Cristi, Santiago Rodríguez y Valverde). En esta región, también existe un gran potencial para desarrollar actividades turísticas en contacto con la naturaleza y la cultura (Orgaz-Agüera y López-Guzmán, 2015; Moral Cuadra et al., 2018), existiendo recursos como las playas, áreas protegidas, el Mercado Binacional de República Dominicana y Haití, gastronomía típica de la región fronteriza, entre otros. Por tanto, y dadas las características de la región, el agroturismo puede ser una actividad que contribuya al desarrollo sostenible y a la reducción de la pobreza.

El agroturismo es una actividad que puede ayudar a los agricultores y a los ganaderos, debido a que a través del turismo pueden obtener mayores recursos económicos. También, cualquier actividad turística ejercida en un área específica puede contribuir al desarrollo de nuevas empresas y a la generación de nuevos empleos (Li et al., 2018), incluso en la actividad agroturística (Mura y Ključnikov, 2018). Por tanto, el agroturismo se configura como una oportunidad para mejorar el desarrollo socioeconómico de la región noroeste de la República 
Dominicana. Si bien, se ha demostrado que es preciso analizar la percepción de los residentes y su relación con el apoyo al desarrollo del turismo antes de desarrollar actividades turísticas. Para ello, esta investigación tenía como objetivo analizar las percepciones de los residentes y su apoyo al desarrollo del agroturismo.

Se ha planteado un modelo para medir las percepciones de los residentes frente a su apego a la comunidad, a su actitud hacia el turismo y a su actitud hacia los turistas. Estas tres variables se han relacionado con el apoyo de los residentes hacia el desarrollo del agroturismo. También, se ha analizado la relación existente entre el conocimiento de los residentes sobre turismo, la confianza en el gobierno local y el apoyo hacia el desarrollo del agroturismo en la región noroeste de la República Dominicana. Por tanto, el presente trabajo ha contribuido, mediante un análisis cuantitativo, a conocer si las comunidades locales de la región noroeste apoyan el desarrollo del agroturismo.

Se ha confirmado una influencia positiva del apego a la comunidad, de la actitud hacia el turismo y de la actitud hacia los turistas sobre el apoyo al desarrollo del agroturismo. Estos hallazgos respaldan los resultados de otros autores (Dyer et al. 2007, Nunkoo y Gursoy, 2012; Vargas et al., 2015; Wang y Xu, 2015; Martín et al., 2015; Moghavvemi et al., 2017). En este sentido, el análisis realizado sobre los residentes que habitan en la región noroeste de la República Dominicana muestra que las personas con cierto apego a su comunidad, con actitudes positivas hacia el turismo y con actitudes positivas hacia los turistas valoran positivamente el desarrollo del agroturismo en la región. Estos resultados positivos pueden deberse a una cierta conciencia de los residentes en favor de la explotación sostenible de los recursos de su comunidad, así como a la búsqueda de nuevas actividades económicas para impulsar el desarrollo de la región.

También se ha confirmado una influencia positiva del conocimiento del turismo por parte de los residentes sobre la confianza en el gobierno local. Estos resultados confirman los hallazgos obtenidos por Latip et al. (2018). En este sentido, un alto conocimiento de los residentes sobre el turismo fomenta la confianza de los residentes hacia el gobierno local y las políticas turísticas. Por lo tanto, las autoridades gubernamentales y locales deben concentrarse en mejorar el conocimiento y la conciencia de los residentes para aumentar su apoyo al desarrollo del agroturismo. En otros estudios, también se ha demostrado que un alto conocimiento sobre turismo de las comunidades locales incrementa su nivel de participación en la actividad turística y su grado de compromiso en la protección y conservación del medio ambiente y los recursos naturales (Latip et al., 2018). Por otro lado, no se ha confirmado que exista una influencia positiva de la confianza en el gobierno local sobre el apoyo al desarrollo del agroturismo. Estos resultados contradicen los hallazgos de Nunkoo y Ramkissoon (2011) y de Nunkoo y So (2015). Este hallazgo sugiere que se puede apoyar el desarrollo de la actividad agroturística independientemente del nivel de confianza que los residentes tengan sobre las políticas turísticas que establece el gobierno local, debido a que también se ha demostrado en otras investigaciones (Cànoves et al., 2006) que existe dificultad para establecer un equilibro entre la actividad turística rural, como puede ser el agroturismo, y la implementación sostenible de las políticas públicas.

A nivel académico, esta investigación ha realizado diferentes aportes. En primer lugar, este trabajo ha revelado algunos de los antecedentes para el apoyo de los residentes hacia el desarrollo del agroturismo. Así, se comprobó que el apego a la comunidad, la actitud hacia el turismo y la actitud hacia los turistas anteceden al apoyo hacia el desarrollo del agroturismo. 
En segundo lugar, esta investigación reveló que es complejo desarrollar la actividad turística sin el apoyo de la comunidad local. Así, se obtuvo que el apego a la comunidad es un elemento importante para explicar el apoyo de los residentes hacia el desarrollo del agroturismo, y que el conocimiento de los residentes frente al turismo es un antecedente importante para determinar su confianza en el gobierno local. Desde el punto de vista práctico, este estudio ha aportado nuevos conocimientos sobre los antecedentes de apoyo al desarrollo del agroturismo, debido a que estas variables no se habían estudiado específicamente en este tipo de turismo. Por tanto, los resultados obtenidos pueden ayudar a los diferentes organismos de gestión del turismo y de la agroindustria.

Los resultados de esta investigación son pertinentes para instituciones públicas que tienen competencia en la gestión del turismo y de la agroindustria, como el Ministerio de Turismo, el Ministerio de Medio Ambiente y Recursos Naturales, y el Ministerio de Agricultura. Hasta la fecha, no existen datos precisos sobre el apoyo de los residentes hacia el agroturismo, y dado que esta investigación ha comprobado que son positivos, podrían servir de base para el desarrollo de estrategias de apoyo al agroturismo como un método para reducir la pobreza (Croes, 2014). Algunas de estas estrategias deben incluir la implicación de los agricultores y ganaderos en la actividad turística, la formación en turismo y desarrollo sostenible de las comunidades locales, la creación de empleo para los residentes en la región y la creación de un marco legislativo del agroturismo.

Este estudio tiene limitaciones y, por lo tanto, los resultados deben interpretarse con precaución. La recolección de datos se basó en muestras de una sola región de República Dominicana y el modelo de investigación fue relativamente simple, ya que no incluyó otros constructos que desencadenan interacciones directas con el apoyo hacia el desarrollo del agroturismo. A pesar de estas limitaciones, el modelo probado genera resultados óptimos para conocer las percepciones de los residentes hacia el apoyo de la actividad agroturística y ofrece oportunidades para futuras investigaciones sobre la relación de otros constructos involucrados en el apoyo de esta actividad turística. El modelo propuesto debería probarse en diferentes contextos geográficos del país en futuras investigaciones, además de indagar en la percepción e interés que la demanda turística tiene sobre el agroturismo.

\section{REFERENCIAS BIBLIOGRÁFICAS}

Agencia EFE (2020). El turismo en tiempos de COVID-19, "un desastre" para todo el Caribe. Recuperado de https://www.efe.com/efe/america/economia/el-turismo-en-tiemposde-covid-19-un-desastre-para-todo-caribe/20000011-4334493

Ali, F., Rasoolimanesh, S.M., Sarstedt, M., Ringle, C.M., y Ryu, K. (2018). An assessment of the use of partial least squares structural equation modeling (PLS-SEM) in hospitality research. International Journal of Contemporary Hospitality Management, 30, (1), 514538.

Andereck, K.L., Valentine, K.L., Knopf, R.C., y Vogt, C.A. (2005). Residents' Perceptions of Community Tourism Impacts. Annals of Tourism Research, 32(4), 1056-1076.

Banco Central (2020a). Estadísticas turísticas 2019. Santo Domingo: Banco Central de la República Dominicana. 
Banco Central (2020b). Resultados Preliminares de la Economía Dominicana, Enero-Diciembre 2019. Santo Domingo: Banco Central de la República Dominicana.

Bianchi, R. (2018). The political economy of tourism development: A critical review. Annals of Tourism Research, 70, 88-102.

Cànoves Valiente, G., Blanco-Romero, A., y Díaz-Soria, I. (2020). Turismo de interior en España, del overtourism al undertourism. Societat d'Història Natural de les Balears, 31, 471482.

Cànoves Valiente, G., Villarino, M., \& Herrera, L. (2006). Políticas públicas, turismo rural y sostenibilidad: difícil equilibrio. Boletín de la Asociación de Geógrafos Españoles, 41, 199-217.

Chin, W.W. (1998). The partial least squares approach to structural equation modeling. Modern Methods for Business Research, 295(2), 295-336.

Clarke, J. (1999). Marketing Structures for Farm Tourism: Beyond the Individual Provider of Rural Tourism. Journal of Sustainable Tourism, 7(1), 26-47.

Cook, K.S., Cheshire, C. Rice, E.R., y Nakagawa, S. (2013). Social Exchange Theory. Dordrecht: Springer.

Cors Iglesias, M. (2020). Una aproximación geográfico-jurídica al agroturismo en España desde el punto de vista de la oferta. Cuadernos de Turismo, 46, 25-46.

Croes, R. (2014). The role of tourism in poverty reduction: An empirical assessment. Tourism Economics, 20(2), 207-226.

Di Domenico, M.L., y Miller, G. (2012). Farming and tourism enterprise: experiential authenticity in the diversification of independent small-scale family farming. Tourism Management, 33(2), 285-294.

Diamantopoulos, A., y Siguaw, J.A. (2006). Formative versus reflective indicators in organizational measure development: A comparison and empirical illustration. British Journal of Management, 17(4), 263-282.

Diamantopoulos, A., y Winklhofer, H.M. (2001). Index construction with formative indicators: An alternative to scale development. Journal of Marketing Research, 38(2), 269-277.

Dijkstra, T.K., y Henseler, J. (2015). Consistent and asymptotically normal PLS estimators for linear structural equations. Computational Statistics \& Data Analysis, 81, 10-23.

Durrande-Moreau, A., Courvoisier, F.H., y Bocquet, A.M. (2017). Le nouvel agritourisme intégré, une tendance du tourisme durable. Téoros, Revue de Recherche en Tourisme, $36(1), 62-74$.

Dyer, P., Gursoy, D., Sharma, B., y Carter, J. (2007). Structural modeling of resident perceptions of tourism and associated development on the Sunshine Coast, Australia. Tourism Management, 28(2), 409-422.

Eusébio, C., Vieira, A.L., y Lima, S. (2018). Place attachment, host-tourist interactions, and residents' attitudes towards tourism development: The case of Boa Vista Island in Cape Verde. Journal of Sustainable Tourism, 26(6), 890-909. 
Fornell, C., y Larcker, D.F. (1981). Evaluating structural equation models with unobservable variables and measurement error. Journal of Marketing Research, 18(1), 39-50.

Gannon, M., Rasoolimanesh, S.M., y Taheri, B. (2020). Assessing the mediating role of residents' perceptions toward tourism development. Journal of Travel Research, 60(1), 149-171.

Grimmelikhuijsen, S. (2012). Linking Transparency, Knowledge and Citizen Trust in Government: An Experiment. International Review of Administrative Sciences, 78(1), 50-73.

Gursoy, D., y Kendall, K.W. (2006). Hosting mega events: Modelling locals' support. Annals of Tourism Research, 33(3), 603-623.

Hair Jr, J.F., Matthews, L.M., Matthews, R.L., y Sarstedt, M. (2017). PLS-SEM or CB-SEM: updated guidelines on which method to use. International Journal of Multivariate Data Analysis, 1(2), 107-123.

Hair Jr, J.F., Sarstedt, M., Hopkins, L., y Kuppelwieser, V.G. (2014). Partial least squares structural equation modeling (PLS-SEM). European Business Review, 26(2), 106-121.

Henseler, J. (2017). Partial least squares path modeling. Cham: Springer.

Henseler, J. (2018). Partial least squares path modeling: Quo vadis?. Quality \& Quantity, 52(1), 1-8.

Henseler, J., Ringle, C.M., y Sarstedt, M. (2015). A new criterion for assessing discriminant validity in variance-based structural equation modeling. Journal of the Academy of Marketing Science, 43(1), 115-135.

Henseler, J., Ringle, C.M., y Sinkovics, R.R. (2009). The use of partial least squares path modeling in international marketing. Bingley: Emerald Group Publishing Limited.

Hetherington, M.J., y Husser, J.A. (2012). How Trust Matters: The Changing Political Relevance of Political Trust. American Journal of Political Science, 56(2), 312-25.

Hu, L. T., y Bentler, P. M. (1998). Fit indices in covariance structure modeling: Sensitivity to underparameterized model misspecification. Psychological methods, 3(4), 424.

Li, K.X., Jin, M., y Shi, W. (2018). Tourism as an important impetus to promoting economic growth: A critical review. Tourism Management Perspectives, 26, 135-142.

Kline, R.B. (2011). Principles and practice of structural equation modeling. New York: Guilford.

Koufteros, X.A. (1999). Testing a model of pull production: a paradigm for manufacturing research using structural equation modeling. Journal of Operations Management, 17(4), 467-488.

Latip, N.A., Rasoolimanesh, S.M., Jaafar, M., Marzuki, A., y Umar, M.U. (2018). Indigenous participation in conservation and tourism development: $A$ case of native people of Sabah, Malaysia. International Journal of Tourism Research, 20(3), 400-409.

Látková, P., y Vogt, C. (2012). Residents' attitudes toward existing and future tourism development in rural communities. Journal of Travel Research, 51(1), 50-67. 
Lee, T.H. (2013). Influence analysis of community resident support for sustainable tourism development. Tourism Management, 34, 37-46.

López, M.F.B., Virto, N.R., Manzano, J.A., y Miranda, J.G.M. (2018). Residents' attitude as determinant of tourism sustainability: The case of Trujillo. Journal of Hospitality and Tourism Management, 35, 36-45.

Martín, H. S., De Los Salmones Sanchez, M.M.G., y Herrero, Á. (2018). Residents' attitudes and behavioural support for tourism in host communities. Journal of Travel \& Tourism Marketing, 35(2), 231-243.

Mccool, S.F., y Martin, S.R. (1994). Community attachment and attitudes toward tourism development. Journal of Travel Research, 32(3), 29-34.

Moghavvemi, S., Woosnam, K.M., Paramanathan, T., Musa, G., y Hamzah, A., (2017). The effect of residents' personality, emotional solidarity, and community commitment on support for tourism development. Tourism Management, 63, 242-254.

Moral-Cuadra, S., Orgaz-Agüera, F., y Maria Dominguez-Valerio, C. (2018). El turismo fronterizo en República Dominicana: análisis de las potencialidades de la frontera dominico-haitiana.Turismo-Estudos e Praticas, 7(1), 22-43.

Moscardo, G. (2005). Peripheral Tourism Development: Challenges, Issues and Success Factors. Tourism Recreation Research, 30(1), 27-43.

Mura, L., y Ključnikov, A. (2018). Small businesses in rural tourism and agro tourism: Study from Slovakia. Economics \& Sociology, 11(3), 286-300.

Nicholas, L.N., Thapa, B., y Ko, Y.J. (2009). Residents' perfectives of a world heritage site: The Pitons Management Area, St. Lucia. Annals of Tourism Research, 36(3), 390-412.

Nunkoo, R. (2015). Tourism development and trust in local government. Tourism Management, 46, 623-634.

Nunkoo, R., y Gursoy, D. (2012). Residents' support for tourism an identity perspective. Annals of Tourism Research, 39(1), 243-268.

Nunkoo, R., y So, K.K.F. (2016). Residents' support for tourism: Testing alternative structural models. Journal of Travel Research, 55(7), 847-861.

Nunkoo, R., y Ramkissoon, H. (2011). Developing a Community Support Model for Tourism. Annals of Tourism Research, 38(3), 964-988.

Nunnally, J.C., y Bernstein, I.H. (1994). Psychometric theory. New York: McGraw-Hill.

OMT (2020). El turismo retrocede a niveles de 1990 con una caída en llegadas del más del 70\%. Madrid: Organización Mundial del Turismo.

ONE (2020a). Tasa de pobreza monetaria general y extrema por regiones de desarrollo, 20002019. Santo Domingo: Oficina Nacional de Estadística de República Dominicana.

ONE (2020b). Censos. Recuperado de https://www.one.gob.do/censos

Orgaz-Agüera, F., y López-Guzmán, T. (2015). Análisis del perfil, motivaciones, y valoraciones de los turistas gastronómicos. El caso de la República Dominicana. ARA: Revista de Investigación en Turismo, 5(1), 43-52. 
Pulido Fernández, J.I. (2008). El turismo rural. Madrid: Síntesis.

Rasoolimanesh, S.M., Taheri, B., Gannon, M., Vafaei-Zadeh, A., y Hanifah, H. (2019). Does living in the vicinity of heritage tourism sites influence residents' perceptions and attitudes?. Journal of Sustainable Tourism, 27(9), 1295-1317.

Rasoolimanesh, S., Jaafar, M., Kock, N., y Ramayah, T. (2015). A revised framework of social exchange theory to investigate the factors influencing residents' perceptions. Tourism Management Perspectives, 16, 335-345.

Rasoolimanesh, S.M., Jaafar, M., Kock, N., y Ahmad, A.G. (2017). The effects of community factors on residents' perceptions toward World Heritage Site inscription and sustainable tourism development. Journal of Sustainable Tourism, 25(2), 198-216.

Real-Aquino, H. (2014). Evolución y Relevancia del Turismo en la República Dominicana. Anuario Jóvenes Investigadores, 1, 75-77.

Roberts, N., y Thatcher, J. (2009). Conceptualizing and testing formative constructs: Tutorial and annotated example. ACM SIGMIS Database: the DATABASE for Advances in Information Systems, 40(3), 9-39.

Rousseau, D. M., Sitkin, S.B., Burt, R.S., y Camerer, C. (1998). Not So Different after All: A CrossDiscipline View of Trust. Academy of Management Review, 23(3), 393-404.

Sarstedt, M., Ringle, C.M., Cheah, J.H., Ting, H., Moisescu, O.I., y Radomir, L. (2020). Structural model robustness checks in PLS-SEM. Tourism Economics, 26(4), 531-554.

Scheyvens, R. (2007). Exploring the Tourism-Poverty Nexus. Current Issues in Tourism, 10(2/3), 231-54.

Sharpley, R. (2008). Tourism, tourists and society. Huntingdon: Elm Publications.

Sharpley, R. (2014). Host perceptions of tourism: A review of the research. Tourism Management, 42, 37-49.

Sheldon, P., y Abenoja, T. (2001). Resident attitudes in a mature destination: The case of Waikiki. Tourism Management, 22(5), 435-443.

Streukens, S., y Leroi-Werelds, S. (2016). Bootstrapping and PLS-SEM: A step-by-step guide to get more out of your bootstrap results. European Management Journal, 34(6), 618632.

Tew, C. y Barbieri, C. (2012). The perceived benefits of agritourism: The provider's perspective. Tourism Management, 33(1), 215-224.

Vargas, A., Oom, P., Da Costa Mendes, J., y Silva, J. (2015). Residents' attitude and level of destination development: An international comparison. Tourism Management, 48, 199-210.

Vargas, A., Plaza, M., y Porra, N. (2009). Understanding residents' attitudes toward the development of industrial tour-ism in a former mining community. Journal of Travel Research, 47(3), 373-387.

Wang, C., Liu, J., Wei, L., y Zhang, T. (2020). Impact of tourist experience on memorability and authenticity: a study of creative tourism. Journal of Travel \& Tourism Marketing, 37(1), 48-63. 
Wang, S., y Xu, H. (2015). Influence of place-based senses of distinctiveness, continuity, selfesteem and self-efficacy on residents' attitudes toward tourism. Tourism Management, 47, 241-250.

Williams, C.C. (2020). Impacts of the coronavirus pandemic on Europe's tourism industry: Addressing tourism enterprises and workers in the undeclared economy. International Journal of Tourism Research, 23(1), 79-88.

Winters, P., Corral, L., y Mora, A.M. (2013). Assessing the role of tourism in poverty alleviation: a research agenda. Development Policy Review, 31(2), 177-202.

\section{CONTRIBUCCIONES DE LOS AUTORES:}

Autor 1: ha sido el responsable de desarrollar el trabajo de campo cuantitativo, además de elaborar la introducción y las conclusiones.

Autor 2: ha sido el responsable de diseñar el instrumento metodológico y elaborar el marco teórico de la investigación, así como la metodología y la tabulación de los datos. Finalmente, se encargó de elaborar el borrador final de artículo, a partir de los apartados realizados por los demás autores.

Autor 3: ha sido el responsable de realizar los análisis estadísticos y desarrollar los resultados, así como parte de las conclusiones.

\section{AGRADECIMIENTOS:}

Los autores de este trabajo quieren agradecer a la Asociación Universitaria Iberoamericana de Postgrado (AUIP) quien financió la estadía del autor Salvador Moral Cuadra en la República Dominicana para diseñar el estudio. También, a la Universidad Tecnológica de Santiago, UTESA Recinto Mao, quienes facilitaron los recursos para desarrollar el trabajo de campo. 